\title{
Habla privada en resolución de problemas en adolescentes de 15 años
}

\section{Fala privada na resolução de problemas em adolescentes de 15 anos Private speech in solving problem with
adolescents 15 years old}

\author{
Cecilia Hontou, Karen Moriera \\ Universidad de la República, Uruguay
}

Autor referente: ceci.hontou@gmail.com

Historia Editorial

Recibido: 17/04/2016

Aceptado: 06/02/2017

\section{RESUMEN}

El fenómeno de hablarse a sí mismo ha sido investigado desde diferentes perspectivas psicológicas. La psicología socio-cultural lo denomina Habla Privada (HP) y lo define como una manifestación audible de lenguaje, autodirigida, con funciones de autorregulación. EI HP permite orientar, monitorear y evaluar la acción. Aparece frente a una demanda cognitiva y sirve para guiar la resolución de problemas. Aunque la mayoría de las investigaciones sobre HP se han centrado en la infancia, existen estudios en adolescentes y adultos que sugieren que el HP continúa siendo una estrategia cognitiva empleada en todo el ciclo vital.

Nuestro objetivo fue estudiar el HP en adolescentes y su relación con la resolución de problemas. Para ello,

aplicamos la Tarea de Formación de Conceptos Artificiales VygotskiSaharov (TFCA) a 19 adolescentes de 15 años, de dos contextos socioeconómicos diferentes, generando protocolos verbales y realizando un análisis microgenético de los mismos.

Se identificó HP en 17 de los 19 adolescentes y se encontró una correlación directa y grande entre HPdesempeño en la tarea. Los adolescentes que tuvieron una puntuación más alta en la tarea emplearon además, más habla privada antecediendo a la acción y más evaluaciones de la acción que sus pares con una puntuación intermedia. Estos resultados aportan evidencia a la hipótesis vigotskiana sobre la relación entre lenguaje y pensamiento.

Palabras clave: Habla Privada, Resolución de problemas, Lenguaje y Pensamiento. 


\section{RESUMO}

O fenómeno de falar com o próprio tem sido estudado a partir de diferentes perspectivas psicológicas. $\mathrm{Na}$ psicologia sociocultural é chamado de Fala Privada (FP) e consiste na manifestação audível da linguagem, autodirigida e com funções de auto regulação. A FP permite a orientação, monitorização e avaliação da ação. Aparece devido a uma demanda cognitiva e serve para guiar na resolução de problemas. Ainda que a maioria das investigações acerca da FP se tenha centrado na infância, existem também estudos com adolescentes e adultos que sugerem que a FP continua sendo uma estratégia cognitiva utilizada ao longo de todo o ciclo vital.

O nosso objetivo foi estudar a FP em adolescentes e a sua relação com a resolução de problemas. Para tal, foi utilizada a Tarefa de Formação de Conceitos Artificiais Vygotski-Saharov (TFCA) em 19 adolescentes de 15 anos, provenientes de dois contextos socioeconómicos, gerando protocolos verbais e realizando uma análise microgenética dos mesmos.

Foi identificada FP em 17 dos 19 adolescentes e encontrou-se uma correlação direita e grande entre FPRendimento na Tarefa. Os adolescentes com pontuação mais elevada na tarefa, empregaram também mais fala privada antecedendo à ação e mais evaluações da ação do que os seus pares com uma pontuação intermédia. Estes resultados aportam evidência às hipóteses Vigotskianas acerca da relação entre a linguagem e o pensamento.

Palavras-chave: Fala Privada, Resolução de Problemas, Linguagem e Pensamento.

\section{ABSTRACT}

The phenomenon of self-addressed speech has been researched from different psychological standpoints. Socio-cultural psychology refers to this as Private Speech (PS). It is defined as an external manifestation of language, which is self-directed and has a selfregulatory function.

PS enables individuals to guide, monitor and evaluate ongoing activities. It appears in moments when people are faced with tasks which demand a high level of cognitive challenge. This is why the relation between PS and problemsolving performance has been researched. Although most of the investigations focus on childhood, there are studies which were carried out by teenagers and adults. Such studies show that PS is a cognitive strategy, which is present throughout the lifespan.

The aim of this article is to present the results of a study which focused on PS in teenagers and its relation with the ability to solve problems. To achieve this, the Vygotsky-Saharov Artificial Concept Formation Task (ACFT) was carried out by nineteen 15-year-old teenagers, from two different socioeconomic contexts. In addition to this, creating verbal protocols and performing a microgenetic analysis thereof.

Findings add to prior evidence regarding the relation between thought and language since seventeen of nineteen teenagers used PS and showed a significant positive correlation between the use of PS and task performance. 


\section{Introducción}

U

na de las claves para comprender la cognición humana ha sido, comúnmente, el lenguaje, por ser exclusivamente humano (Gomila, 2012). La relación lenguaje y pensamiento es ampliamente estudiada y discutida en la historia de la psicología.

El estudio de la relación entre lenguaje y pensamiento continúa vigente hoy (Gomila, 2012). Por ejemplo, en ciertas ocasiones las personas se hablan a sí mismas, presentan vocalizaciones externas, audibles para un tercero pero sin finalidad comunicativa (Berk, 1992; Winsler, 2009). Este fenómeno ha sido denominado de diferentes maneras según el marco teórico que lo aborda, en este artículo utilizaremos el término Habla Privada (HP).

\section{Habla privada desde la perspectiva socio-cultural}

Vygotski (1995) identificó los fenómenos de HP bajo la denominación de habla egocéntrica y planteo que este tipo de habla "se convierte en instrumento del pensamiento propiamente dicho, en la búsqueda y planificación de la solución a un problema" (p. 71). En su perspectiva, el HP influye en procesos cognitivos superiores, tales como: la atención selectiva, la memoria voluntaria, la planificación, la formación de conceptos y la auto-reflexión (Berk, 1992).

EI HP aparece como una función fisiológicamente externa pero con orientación interna, de autorregulación. Esta definición pone de relieve el origen social del HP. En un primer momento la regulación es dada verbalmente por el cuidador del niño y luego éste comienza a utilizar el HP para autorregular su conducta. EI HP es un ejemplo de la interiorización del lenguaje, en tanto mediador del pensamiento. Se observa en la transición entre el habla externa al habla interna, presentando un desarrollo curvilineal, 
surgiendo en la edad preescolar, incrementándose hasta los 5-6 años y disminuyendo luego su frecuencia hasta interiorizarse completamente.

El proceso de interiorización del habla ha llevado a los investigadores a enfocarse en su desarrollo, uso y función entre los 4 y 9 años (Sánchez-Medina, Alarcón-Rubio, \& Mata-Benítez, 2009; Winsler \& Naglieri, 2003), aunque también existen investigaciones que registraron HP después de los 9 años, en la adolescencia y la adultez (JohnSteiner, 1992; Kronk, 1994; Winsler \& Naglieri, 2003).

Para varios autores (Duncan \& Tarulli, 2009; Kronk, 1994) a medida que se avanza en el desarrollo el HP decrece debido a presiones sociales, por lo que los adolescentes y adultos se vuelven reticentes a ser observados utilizando HP. Desde la etapa escolar se va incorporando esta noción, por ejemplo en el salón de clase las maestras desaprueban este tipo de habla (Deniz, 2009; Frauenglass \& Diaz, 1985; Manfra \& Winsler, 2006). Otro motivo por el que el HP no es socialmente aceptada es que las personas pueden asociarla con signos de psicopatología (Duncan \& Tarulli, 2009)

A pesar del desarrollo curvilineal del HP, su presencia en adolescentes y adultos es consistente con el planteo socio-cultural, ya que el mismo Vygotski (2000) establece como condición de aparición una perturbación del curso normal de la actividad, en "la aparición de las dificultades entran en juego los medios auxiliares; esta es la regla general que podemos deducir de todos nuestros experimentos" (p. 241). Entonces la presencia de HP resurge luego de ser interiorizada en momentos de alta demanda cognitiva, frente a la necesidad de completar una tarea difícil o nueva, independientemente del momento evolutivo (Alarcón-Rubio, Sánchez-Medina, \& Winsler, 2013).

El hecho de que el HP surja para realizar una tarea difícil, supone a la misma como un instrumento semiótico que se utiliza para lograr competencia en actividades cognitivamente demandantes. De esta forma, se postula la influencia de ésta en el pensamiento, siendo un medio en la resolución de problemas (Duncan \& Cheyne, 
2001). Por consiguiente, se vuelve foco de interés la relación entre HP y el desempeño en la resolución de problemas.

\section{Estudios sobre habla privada en adolescentes y adultos}

Winsler y Naglieri (2003), realizaron un estudio a gran escala con niños de entre 5 y 17 años con el objetivo de analizar la hipótesis vygotskiana del desarrollo curvilineal del habla en tareas perceptuales. Reportaron el uso de HP en todos los niveles de edad, con una disminución marcada a partir de los 9 años. Los autores concluyen que el HP continúa siendo una herramienta cognitiva para la resolución de problemas y una estrategia durante la infancia media y la adolescencia, aunque no encuentran relaciones entre HP y desempeño para niños mayores de 6 años.

El primer estudio enfocado exclusivamente en adolescentes fue el de Kronk (1994), que planteó la hipótesis de que la disminución en los niveles de producción de HP podrían derivar de la sanción social del hablarse a sí mismo en forma explícita. Para probar esto incluyó en un estudio a 47 adolescentes que tomaron un examen de 30 minutos en un salón con cómplices entrenados para utilizar HP luego de los primeros 10 minutos. En esa situación 46 de los 47 adolescentes presentaron HP, aunque no se encontró correlación entre HP y desempeño en el examen.

Duncan \& Cheyne (2001) en un estudio con universitarios (53 participantes), registraron HP mientras estos resolvían tareas manuales. Los autores concluyeron que el HP continuaba teniendo un rol mediador en la resolución de problemas y en procesos de auto-regulación en la adultez. A pesar de ello, tampoco hallaron correlación entre HP-desempeño, salvo algunas excepciones sujetas a los niveles de dificultad.

Alarcón-Rubio et al. (2013) realizaron un estudio con adultos que asistían a un programa de alfabetización, aplicando una tarea semántica de clasificación de objetos. 
Registraron HP y encontraron una correlación positiva entre HP auto-reguladora y desempeño y HP que precede la acción y desempeño.

Investigaciones relativas a la adquisición de nuevas competencias, muestran de manera sistemática el recurso al HP como estrategia cognitiva (John-Steiner, 1992) lo que es consistente con el planteo vygotskiano sobre el rol mediador del habla en la consolidación del dominio de habilidades y funciones (Sánchez-Medina et al., 2009).

\section{Problemas metodológicos en el estudio del Habla privada}

Los estudios presentados han demostrado que el HP en la adolescencia y la adultez está presente y desempeña funciones cognitivas, pero no son concluyentes en cuanto a la relación de HP y desempeño. Sobre esta relación se ha señalado un patrón confuso de resultados, que se debería a limitaciones metodológicas en los estudios (Diaz, 1992; Frauenglass \& Diaz, 1985; Montero \& De Dios, 2006; Winsler, Diaz, \& Montero, 1997). Particularmente el HP aparece ante tareas con alta demanda cognitiva, por lo que, en primer lugar, no es esperable que aparezca si la tarea puede resolverse en forma automática. En segundo lugar, y más importante, el HP aparecería frente a las dificultades con lo cual, si la tarea fuera extremadamente difícil debería correlacionarse más con el fracaso que con el éxito (Frauenglass \& Diaz, 1985). De este modo, testear la hipótesis de que el uso del HP conduce a mejoras en el desempeño implica identificar un rango de dificultad específico. El HP conduciría a la mejora en el desempeño sólo en los casos en los que la dificultad de las capacidades del sujeto.

Por todo lo expuesto resulta relevante estudiar la relación entre HP y desempeño particularmente en la adolescencia, donde existen pocos estudios y donde la restricción metodológica sobre el grado de dificultad de la tarea no siempre ha sido contemplada. 
El presente trabajo se propuso estudiar la relación entre HP y desempeño en adolescentes de 15 años considerando una tarea con el nivel de dificultad apropiado para la edad, en el sentido de resultar desafiante y al mismo tiempo estar dentro de las posibilidades de resolución del sujeto (Diaz, 1992).

A tales efectos interesó testear la idoneidad de la Tarea de Formación de Conceptos Artificiales Vygotski-Saharov (TFCA) (Hanfmann \& Kasanin, 1937, 1942) para elicitar procesos de HP.

Las hipótesis del estudio fueron que (a) los adolescentes utilizarían HP como estrategia para resolver la TFCA, (b) aquellos que utilizaran más HP tendrían mejor desempeño, (c) el habla privada que precediera la acción estaría relacionada positivamente con un mejor desempeño (revelando una función de planificación).

\section{Método}

\section{Participantes}

19 adolescentes de 15 años (media de 15,8; DS $=.27$ ), 9 hombres $(47 \%$ ), que cursaban $4^{\circ}$ año de liceo. Los sujetos provenían de dos centros educativos públicos de la ciudad de Montevideo, 10 de un centro educativo de nivel socio-económico medio y 9 de nivel socio-económico bajo. La muestra quedó conformada por todos los adolescentes que entregaron el consentimiento informado de los responsables legales en ambos centros. Como criterio de exclusión se consideró la repetición de algún año del cico educativo. En todos los casos se solicitó consentimiento informado de las familias, teniendo la posibilidad de retirarse del estudio por su propia voluntad, asegurando la confidencialidad y el resguardo de los datos. A su vez, se aseguró la completa confidencialidad de la identidad de los participantes.

\section{Diseño}


La metodología del estudio combinó análisis cuantitativo y cualitativo. El desempeño en la tarea se midió con la escala subjetiva de Hanfmann y Kasanin $(1937,1942)$ y para el HP se generó un protocolo verbal que se analizó microgenéticamente (Smagorinsky, 1998). Debido a que los tamaños muéstrales fueron pequeños (19 sujetos) y que la distribución de las variables no fue normal se trabajó con pruebas estadísticas no paramétricas. Los análisis estadísticos realizados fueron la mediana, el percentil 25, el percentil 75 , la correlación de Spearman y la prueba U de MannWhitney para muestras independientes. Se utilizó como nivel de significación $p<.05$ representado por ${ }^{*}$ y $p<.001$ representado por ${ }^{* *}$. Se consideró como nivel de correlación: pequeño $r s \geq .10$, medio $r s \geq .30$, grande rs $\geq .50$.

\section{Instrumentos y procedimiento}

\section{Tarea de Formación de Conceptos Artificiales}

La TFCA es una tarea de clasificación que consiste en formar cuatro clases mutuamente excluyentes, determinadas por la combinación de dos atributos. Es una tarea de resolución progresiva, ya que a medida que avanza el sujeto va obteniendo nueva información para resolverla, gracias a las correcciones realizadas por el entrevistador.

En un tablero dividido en cuatro áreas con un círculo en el centro se presentan al sujeto 22 bloques de madera. Los bloques tienen seis formas (círculo, cuadrado, hexágono, semicírculo, trapecio y triángulo), cinco colores (amarillo, blanco, marrón, negro y verde), dos alturas (altos y bajos) y dos tamaños (grandes y chicos). Cada bloque tiene una etiqueta con una palabra sin sentido de tres letras: los bloques chicos y bajos dicen CEV, los bajos y grandes dicen BIK, los altos y grandes dicen LAG y los altos y chicos dicen MUR.

En un principio se presenta el tablero con los 22 bloques de madera con la etiqueta 
hacia abajo, en el círculo del centro y las cuatro secciones del tablero vacías. Se le enseña la etiqueta de un bloque (triángulo, marrón, alto y chico) colocándolo en una de las secciones del tablero, se le consigna que agrupe aquellos bloques que se parecen a la muestra y se explicita la existencia de cuatro grupos. Luego que el sujeto realiza su primera clasificación, el entrevistador le muestra la etiqueta de una pieza mal colocada, que pone en evidencia que su clasificación era errónea y genera una nueva muestra en otra sección del tablero. A cada nuevo bloque con la etiqueta a la vista se lo denomina corrección. El sujeto probará otra clasificación y el entrevistador continuará corrigiendo bloques hasta que se den vuelta los 21 bloques o alcance la solución correcta. Cada vez que realiza una agrupación, se indaga verbalmente el motivo de la misma.

Una vez alcanzada la solución y explicitados los criterios de agrupación, se pide al sujeto que vuelva a realizar la división en cuatro grupos de los bloques, nuevamente, mezclados sin la etiqueta a la vista en el centro del tablero, a esta instancia se la denomina Repetición (Figura 1).
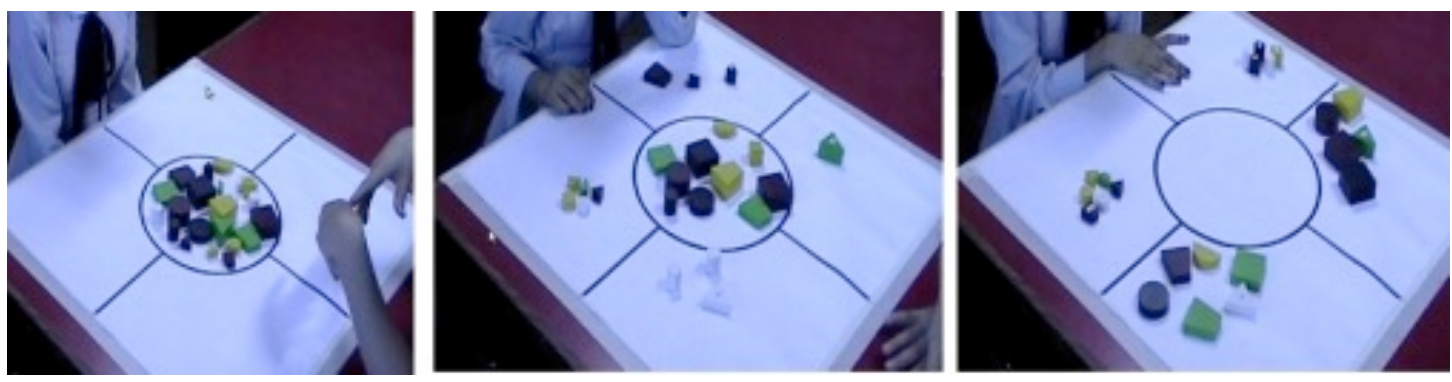

Figura 1. Secuencia de resolución de la TFCA.

\section{Escala Subjetiva Hanfmann y Kasanin}

La escala subjetiva de Hanfmann y Kasanin $(1937,1942)$ se compone por tres secciones: a) Interpretación de las Instrucciones, b) Intentos de Solución y c) Hallazgo y Dominio de la solución correcta. La escala se puntúa del 12 al 36, pudiendo obtener 
un mínimo de 4 y un máximo de 12 por cada sección. A su vez, se subdividió en tres niveles de rendimiento: $12-16$ primitivo, 17-31 intermedio y 32-36 superior. Para una descripción completa de los niveles de desempeño ver Hanfman \& Kasanin (1942).

Cada sujeto fue entrevistado una única vez donde se aplicó la TFCA que fue videograbada con cámara fija. Los datos se desgrabaron en una planilla por sujeto, transcribiendo los enunciados del entrevistador, el entrevistado y la acción del entrevistado en la resolución de la tarea. Los datos se procesaron con el paquete estadístico SPSS 21.

\section{Protocolo verbal}

Se consideró como unidad de análisis al enunciado, definido como cualquier oración completa o fragmentada, o con intenciones marcadas de finalización, los turnos conversacionales o cualquier verbalización que estuviera separada de otra por al menos 2 segundos, también se tomó el criterio de discontinuidad semántica (Winsler, Fernyhough, McClaren, \& Way, 2005). No se consideraron como enunciados aquellas verbalizaciones monosilábicas que respondían a una pregunta del entrevistador.

Se conformó un sistema de categorías para el registro de HP. Los enunciados se clasificaron según Función (intelectual/comunicativa), Dirección (auto-dirigida/heterodirigida) y Grado Espontaneidad (Espontáneo/ No Espontáneo). Como criterio para la identificación de un enunciado como HP requirió que combinara las clasificaciones los atributos de Intelectual/Auto-dirigida/Espontáneo.

Se creó la categoría Movimiento para clasificar aquellos enunciados de HP que precedieran la acción, siguiendo la hipótesis vygotskiana sobre la secuencia evolutiva de las relaciones entre HP y acción, que se inicia con la acción precediendo a la verbalización, y finaliza con la verbalización anticipando y regulando a la acción (Berk, 1992).

Los contenidos del HP fueron tipificados en 7 categorías: Agrupaciones que refiere a 
aquellos enunciados sobre la naturaleza de la agrupación, Materiales referida a enunciados que hacían mención a características de los bloques o estaban relacionados con la consigna de la tarea, Acción atendiendo a las descripciones verbales de la acción del sujeto, Evaluación que refiere a los juicios sobre el desempeño del sujeto o a la tarea en sí misma, Incompletos que refiere a aquellos enunciados que no se podían clasificar el contenido por tratarse de frases incompletas, No asignable refiere a vocalizaciones que no fueron audibles y Otros. 


\section{Tabla 1}

Categorías de Contenido de HP

\section{$\begin{array}{lll}\text { Clasificación } & \text { Definición } & \text { Ejemplo }\end{array}$}

\section{Dirección}

Autodirigido

Enunciados dirigidos al propio sujeto, sin intención comunicativa

Heterodirigido Enunciados dirigidos al interlocutor

Función

Intelectual

Comunicativo

Iniciación

Espontánea

No espontánea

Iniciada por el sujeto, sin estímulo del interlocutor

Refieren a la orientación del pensamiento y/o la acción

Responde a, o plantea demandas al interlocutor

Iniciada a solicitud del interlocutor
Dos triángulos y dos... ¿qué me preguntaste, perdón?

Podría mover todos los de la misma altura para acá y los de la otra altura para ese lado.

Porque me parece que ahora que me revelaste (señala una figura), que podría ser por el color

Tiene que haber otro criterio para cambiarlos

No sé por qué lo hice así, creo que es porque distinto color y distinta forma

(mueve pieza) aunque el tamaño, también, sigue siendo grande

Claro, esta figura es la misma que esta, entonces capaz que coinciden en algo, no sé. Lo que probé es... Ay, cómo te puedo explicar...

\section{Contenido}

Agrupación

Enunciados que explicitan criterios de agrupación posibles (empleados o no)

Materiales

Describen la características de los materiales y/o de la consigna de trabajo

Acción

Descripciones verbales de la acción realizada o a realizar

Evaluación

Juicios sobre el desempeño o sobre la tarea

Incompleto

No asignable
Enunciados que comienzan siendo audibles y comprensibles pero que no completan una idea

Vocalizaciones sólo parcialmente audibles, pero incomprensibles
Capaz que por lo más alto y lo más bajo

no tengo cuatro de estos

(hexágonos), ni cuatro de estos

(semicírculos), pero tengo cuatro de estos (círculos), no cinco. / hay mucha variedad de círculos y formas

Ésta la sacaría de acá. / Voy a sacar estos.

Ahora sí que se me complicó / estoy más cerca pero no

La razón sería... / Capaz que es por... / A no ser que...

Murmura / Mmmm... 


\section{Resultados}

En los resultados no se incluyó contexto como un factor entre los sujetos, ya que no se encontraron diferencias significativas entre los grupos ( $U$ de Mann-Whitney, $p=1.00$ ). No se incluyó sexo como factor, ya que tampoco se encontraron diferencias significativas ( $U$ de Mann-Whitney $p=1.00)$.

Análisis de desempeño en TFCA.

En un primer momento se analizó el desempeño en la tarea, para poder diferenciar grupos de rendimiento. Para los 19 adolescentes la mediana de la escala subjetiva fue de 33 puntos $(27,35)$.

Para analizar la dificultad de la tarea, se utilizó la separación de grupos por nivel de rendimiento, quedaron conformados dos grupos: nivel intermedio y nivel superior; no se ubicó ningún sujeto en el nivel primitivo. El nivel intermedio se compone por 8 sujetos $(42 \%)$ y el superior por 11 sujetos $(58 \%)$.

Con el fin de determinar en qué aspectos se diferenciaban los grupos se analizó, en primer lugar la cantidad de correcciones que necesitaron los sujetos de cada nivel de desempeño y se encontró que fue significativamente diferente para ambos grupos con un mínimo 2 y máximo de 11 entre 21 correcciones posibles $(p<.001)$. Luego se analizaron las diferentes subescalas de la escala subjetiva (Interpretación de las instrucciones, Intentos de Solución y Hallazgo y dominio). Se aplicó la prueba U de Mann-Whitney de muestras independientes y se encontraron diferencias significativas para Interpretación de las instrucciones $(p<.001)$ e Intentos de Solución $(p<.001)$, no así para Hallazgo y dominio $(p=.395)$. Interesó emprender un análisis de los componentes de Interpretación de las instrucciones, a los cuales también se aplicó la prueba U- Mann-Whitney. Se encontraron diferencias significativas en los cuatro componentes de esta subescala: Principio $(p<.001)$, Nombre $(p<.001)$, Muestra $(p=$ 
$.005)$ y Totalidad $(p=.001)$.

Se analizaron las puntuaciones obtenidas por los dos grupos en los componentes de las tres subescalas. El nivel intermedio obtuvo una mediana de 2 puntos para los componentes Principio, Nombre y Muestra y de 1 punto para Totalidad, en cambio el nivel superior obtuvo una mediana de 3 puntos en los cuatro componentes. En Intentos de solución el nivel intermedio obtuvo una mediana de 8 puntos, mientras la del nivel superior fue de 10 puntos. En los cuatro componentes de Hallazgo y Dominio de la solución ambos grupos obtuvieron una mediana de 3 puntos en los cuatro componentes.

En cuanto a Interpretación de las instrucciones los sujetos de nivel intermedio interpretaron la tarea como un juego reglado, donde el nombre de los bloques únicamente establecía los bloques que iban juntos, pudieron valerse de la muestra parcialmente y desatendieron el requerimiento de la agrupación cuádruple. En cambio, los sujetos de nivel superior abordaron la tarea buscando un principio de clasificación, atribuyéndole atributos en común a los nombres, cada corrección (o nueva muestra) los conducía a revisar sus criterios de clasificación y consideraban el requerimiento de agrupación cuádruple. Referido a Intentos de solución, el nivel intermedio se basó en atributos objetivos de los bloques, pero falló en jerarquizar sistemáticamente el mismo atributo. Pudo conformar generalizaciones en base a atributos relevantes, pero no generó categorías excluyentes. El nivel superior conformó clases excluyentes, jerarquizando los atributos relevantes o realizó generalizaciones complementarias (e.j. "una de cada color en cada grupo). En el caso de Hallazgo y dominio ambos grupos llegaron a la solución por insight, pudiendo formular el criterio de agrupación, reconocieron el sistema de doble dicotomía (tamaño y altura) y efectuaron la Repetición correctamente.

\section{Análisis de los protocolos verbales}


Se analizaron 1.647 enunciados, con una mediana por sujeto de 65 enunciados (47, 130). Para el análisis de los enunciados se calculó el porcentaje de enunciados de cada categoría sobre el total de enunciados.

Se registró presencia de HP en 17 de los 19 participantes (90\%), con una mediana del porcentaje de enunciados de HP de 48,81\% (31,58\%, 62,39\%). La mediana del porcentaje de enunciados de HP que preceden la acción (Movimiento) fue de 20,83 \% $(14,08 \%, 31,25 \%)$.

Para la relación entre HP-desempeño se utilizó correlación de Spearman. La tabla 2 presenta estas correlaciones.

Tabla 2

Correlaciones de Spearman para HP-desempeño TFCA

\begin{tabular}{lllcccc}
\hline & \multicolumn{5}{c}{ Rho } & \multicolumn{2}{c}{$\begin{array}{c}\text { Interpretación } \\
\text { Desempeño } \\
\text { Categorias }\end{array}$} & $\begin{array}{c}\text { Intentos } \\
\text { Spearman }\end{array}$ & $\begin{array}{c}\text { Hallazgo y } \\
\text { TFCA }\end{array}$ & $\begin{array}{c}\text { de } \\
\text { Instrucciones }\end{array}$ & $\begin{array}{c}\text { dominio de } \\
\text { solución }\end{array}$ & $\begin{array}{c}\text { Habla } \\
\text { la solución }\end{array}$ & Privada \\
\hline Habla Privada & $\begin{array}{l}\text { Rho } \\
\text { Spearman }\end{array}$ &, $570^{*}$ &, $635^{* *}$ &, $537^{*}$ &, 309 & \\
& $\begin{array}{l}\text { Sig. } \\
\text { (bilateral) }\end{array}$ &, 011 &, 004 &, 018 &, 197 & \\
& $\begin{array}{l}\text { Rho } \\
\text { Spearman }\end{array}$ &, $651^{* *}$ &, $695^{* *}$ &, $589^{* *}$ &, 277 &, $847^{* *}$ \\
$\begin{array}{l}\text { Habla Privada } \\
\text { que precede } \\
\text { la acción }\end{array}$ & $\begin{array}{l}\text { Sig. } \\
\text { (bilateral) }\end{array}$ &, 003 &, 001 &, 008 &, 251 &, 000 \\
\hline
\end{tabular}

Nota. Nivel de significanción ${ }^{*} p<.05 * * p<.001$.

Tal como se desprende de la tabla 2, las correlaciones entre la presencia de HP y de HP que precede a la acción con las variables que evalúan el desempeño efectivo en la tarea fueron positivas y grandes, salvo en el caso de Hallazgo y dominio de la solución, donde no se verifica asociación. 
Para saber si existían diferencias en el uso de HP de acuerdo al nivel de desempeño en la tarea, se realizó una prueba $U$ de Mann Whitney categorizando a los sujetos en dos niveles de desempeño (superior e intermedio). Se encontró que los sujetos que se encontraban en el rango de desempeño superior tuvieron mayor uso de HP $(p=.017)$ y más HP que precedía a la acción $(p=.015)$ que los que se encontraban en el rango de desempeño intermedio.

También se analizó el contenido del HP, teniendo en cuenta únicamente a aquellos sujetos que tuvieron presencia de HP $(\mathrm{N}=17)$. En este caso, se calculó el porcentaje de enunciados de cada categoría sobre el total de enunciados de HP.

Como se mencionó en la tabla 1, las categorías de contenido fueron: agrupación, materiales, acción, evaluación, incompleto y no asignable. A estas categorías se agrega la categoría Otros, que englobó a las emisiones no incluidas en las categorías anteriores.

A continuación se expone la mediana, el percentil 25 y el percentil 75 para las distintas categorías: Agrupación 7,69 \% (4,16\%, 15,86\%), Materiales 10,42\% (5,56\%, 14,81\%), Acción 5,95\% (3,33\%, 12,50\%), Evaluación 2,08\% (0,59\%, 3,33\%), Incompleto 2,08\% $(1,38 \%, 3,79 \%)$, No asignable $5,95 \%(2,81 \%, 7,01 \%)$ y Otros $2,38 \%(0 \%, 4,16 \%)$.

Para la relación entre los distintos tipos de HP y desempeño se aplicó correlación de Spearman, se encontraron relaciones estadísticamente significativas con dos categorías: Incompleto $r s=.496^{*}, p=, 043$ y No asignable $r s=.527^{*}, p=.030$. En la Tabla 2 se pueden observar las correlaciones entre desempeño y todas las categorías de Contenido. 


\section{Tabla 3}

Correlaciones de Spearman para Categorías de Contenido

\begin{tabular}{lccccccc}
\hline $\begin{array}{c}\text { Desempeño en } \\
\text { TFCA }\end{array}$ & Agrupación & Materiales & Acción & Evaluación & Incompleto & $\begin{array}{c}\text { No } \\
\text { asignable }\end{array}$ \\
\hline Rho Spearman &, 244 &, 225 &, 001 &, 422 &, $496^{*}$ &, $527^{*}$ \\
Sig. (bilateral) &, 346 &, 385 &, 996 &, 091 &, 043 &, 030 \\
\hline
\end{tabular}

Nota. Nivel de significación * $p<.05$.

De los 19 adolescentes 17 presentaron HP, los 2 sujetos que no presentaron HP tuvieron un desempeño pobre en la TFCA. Para el análisis cualitativo se consideraron aquellas entrevistas que tenían más que un $20 \%$ de sus enunciados de $\mathrm{HP}$, por el motivo que se consideró bajo el peso relativo de HP en la resolución de la TFCA. De los 4 sujetos que presentaron 3/4 partes de sus enunciados comunicativos, 3 de ellos también tuvieron un desempeño pobre. La mayoría de los adolescentes comenzaron la tarea utilizando HP antes de recibir ninguna corrección. De los 10 que comenzaron con HP, 6 evalúan criterios de agrupación, 2 utilizan HP precede la acción y 2 no es asignable el contenido (murmuraciones). Globalmente más de la mitad de las correcciones fueron seguidas por enunciados de HP, 8 de los 13 sujetos enfrentaron la mayoría de sus correcciones con HP. Se identificaron secuencias de habla que comenzaban dirigidas al entrevistador con una función comunicativa y luego cambiaba su función a intelectual, culminando en el movimiento de una pieza.

\section{Discusión}

El objetivo del presente estudio fue investigar el HP en adolescentes en resolución de problemas y su relación con el desempeño efectivo en la TFCA. 
Respecto al desempeño en la tarea, se pudieron distinguir dos niveles de rendimiento, de lo cual se interpreta que la TFCA es una tarea que se encuentra dentro de la zona de posibilidades de resolución autónoma de los sujetos de este nivel de edad (Moreira, 2012; Towsey, 2009). En este sentido, y considerando los planteos de Diaz (1992) y Montero \& De Dios (2006) la tarea seleccionada tuvo una dificultad acorde para la producción de HP en adolescentes, lo que se reflejó en el hecho de que la mitad de los sujetos requirió al menos 6 correcciones para alcanzar la solución (lo que implica casi 2 muestras de ejemplares positivos por grupo).

El resultado de la presencia de HP adolescentes es consistente con los presentados por Kronk (1994) y Winsler \& Naglieri (2003) para adolescentes. Este resultado también aporta evidencia a la tesis de que el HP sigue siendo una estrategia cognitiva luego de la infancia, más que una fase del desarrollo como había sido inicialmente el planteo de Vygotski. La tesis de Vygotski (1995) se relaciona con el proceso de interiorización del habla, después de los 7-8 años el niño comienza a utilizar el habla interna, pero aun así los sujetos continúan utilizando HP (Duncan \& Tarulli, 2009). El marco propuesto por Wertsch (1991), supone que la coexistencia entre HP y habla interna se debe a la heterogeneidad, en vez de a una jerarquía genética.

Nuestra hipótesis sobre que el HP se relacionaría positivamente con un mejor desempeño en la tarea, recibió apoyo por la correlación positiva y fuerte entre HP y escala subjetiva (donde a mayor uso de HP mejor rendimiento) así como por las diferencias entre el uso de HP de acuerdo con el nivel de desempeño en la tarea. Estos resultados difieren de los de Kronk (1994), quizás porque Kronk no consideró la tarea como una variable en la relación HP-desempeño. Tampoco concuerda con el estudio de Winsler \& Naglieri (2003), en este caso se puede pensar que la discordancia se relaciona con el tipo de tareas aplicadas, pues el estudio de Winsler \& Naglieri (2003) empleó tareas viso-espaciales, mientras el nuestro recurrió a tareas de categorización que requerían la formación de nuevos conceptos, y en este sentido se 
puede pensar que el componente semántico predomina sobre el viso- espacial. Nuestros resultados son consistentes con los de Alarcón-Rubio et al. (2013), que utilizaron una tarea semántica. Para futuras investigaciones, resulta interesante realizar un estudio que compare la relación HP-desempeño en una tarea viso-espacial y en una tarea semántica con el fin de explorar con mayor profundidad la influencia de las tareas.

A su vez, la relación positiva y fuerte de HP-desempeño apoya el planteo de Vygotski (1995) de que el HP se convierte en instrumento del pensamiento, en tanto permite al sujeto alcanzar una resolución exitosa del problema. Este resultado aporta evidencia a la hipótesis de que una de las funciones cognitivas del lenguaje en los procesos de pensamiento es la regulación de la acción, contribuyendo a la eficacia del control ejecutivo.

La cuarta hipótesis de que el HP que precede la acción tenía una relación positiva con el desempeño fue confirmada y concuerda con los resultados de Alarcón-Rubio et al. (2013). Ambos resultados son consistentes con el planteo de Vygotski (1995) de que el HP que precede la acción tiene una función auto-reguladora de planificación y moduladora de la conducta conduciendo a una resolución más exitosa de la tarea.

Se encontraron distintos tipos de HP, las categorías Incompleto y No asignable se relacionaron positivamente con un mejor desempeño. El resultado de la categoría No asignable es coherente con aquellos estudios que utilizan como categoría el grado de encubierto de los enunciados de HP (para una mejor comprensión de la categoría ver Winsler et al., 2005), ya que los enunciados categorizados como No asignable eran murmuraciones, en tanto manifestaciones encubiertas de HP. Este resultado es consistente con los obtenidos por Alarcón Rubio et al. (2013) que mostraron que en adultos iletrados, las manifestaciones parcialmente interiorizadas de HP, contribuyeron, en mayor medida a un buen desempeño, que las completamente externas. Al mismo tiempo es consistente con la hipótesis vygotskiana de la 
internalización del HP. Respecto a la relación directa y moderada entre DesempeñoIncompleto habría que continuar indagando los motivos de esta relación, en un principio nos podemos inclinar por la hipótesis vygotskiana de que a medida que el habla se interioriza se va encriptando y se vuelve menos comprensible para el tercero que le es audible (Vygotski, 1995). Por otra parte, parece interesante indagar sobre la función de estos enunciados en el pensamiento, como hipótesis a indagar se podría pensar en que una posible función puede ser de estructuración del pensamiento en el sentido de establecer un orden: "Entonces...", "Capaz que es por...". Los resultados obtenidos en este estudio aportan evidencia favorable a que el lenguaje influye en el pensamiento, a través de su mediación el sujeto se autorregula, guiando, monitoreando y evaluando su acción. En la adolescencia la mediación del lenguaje continúa influyendo en los procesos de pensamiento, coexistiendo el HP con el habla interna.

Entre las principales limitaciones del estudio se destacan el pequeño tamaño de la muestra, que se relaciona con el enfoque metodológico de análisis microgenético. Más allá de ello sería interesante replicar estos hallazgos con muestras de mayor tamaño. Una segunda limitación es que no fue posible realizar procedimientos de fiabilidad interjueces, dado que los datos ya habían sido analizado por una de las investigadoras en un primer estudio y no se disponía de recursos para codificadores externos.

El estudio muestra que es relevante continuar analizando la relación entre HPdesempeño permitirá estudiar de qué modo el HP puede ser una estrategia en adolescentes, ya que permite al sujeto auto-controlar su conducta, planificar, priorizar, inhibir respuestas prepotentes y supervisar, conjuntamente procesos de las funciones ejecutivas. 


\section{Referencias}

Alarcón-Rubio, D., Sánchez-Medina, J. A., \& Winsler, A. (2013). Private Speech in Illiterate Adults: Cognitive Functions, Task Difficulty, and Literacy. Journal of Adult Development, 20(2), 100-111. doi:10.1007/s10804-013-9161-y

Berk, L. (1992). Children's private speech: An overview of Theory and the status of research. En R. M. Diaz, \& L. E. Berk (Ed.), Private speech: From social interaction to self-regulation (pp. 17-53). New York: Psychology Press Taylor \& Francis Group.

Deniz, C. B. (2009). Early Childhood Teachers' Awareness, Beliefs, and Practices toward Children's Private Speech. En A. Winsler, C. Fernyhough, \& I. Montero (Eds.), Private speech: Executive functioning, and the development of verbal self-regulation (pp. 236-246). New York: Cambridge University Press.

Diaz, R. M. (1992). Methodological concerns in the study of private speech. En R. M. Diaz, \& L. E. Berk (Ed.), Private speech: From social interaction to selfregulation (pp. 17-53). New York: Psychology Press Taylor \& Francis Group.

Duncan, R. M., \& Cheyne, J. A. (2001). Private speech in young adults: Task difficulty, self-regulation, and psychological predication. Cognitive Development, 16(4), 889-906.

Duncan, R. M., \& Tarulli, D. (2009). On the persistence of private speech: Empirical and theoretical consideration. En A. Winsler, C. Fernyhough, \& I. Montero (Eds.), Private speech: Executive functioning, and the development of verbal self-regulation (pp. 176-187). New York: Cambridge University Press.

Frauenglass, M. H., \& Diaz, R. M. (1985). Self-regulatory functions of children's private speech: A critical analysis of recent challenges to Vygotsky's theory. Developmental Psychology, 21, 357-364.

Gomila, A. (2012). Verbal Minds: Language and the architecture of cognition. London: 
Elsevier.

Hanfmann, E., \& Kasanin, J. (1937). A Method for the Study of Concept Formation. The Journal of Psychology, 3(2), 521-540.

Hanfmann, E., \& Kasanin, J. (1942). Conceptual thinking in schizophrenia. New York: Nervous and Mental Disease Monographs.

John-Steiner, V. (1992). Private speech among adults. En R. M. Diaz, \& L. E. Berk (Eds.), Private speech: From social interaction to self-regulation (pp. 285-295). New York: Psychology Press Taylor \& Francis Group.

Kronk, C. (1994). Private speech in adolescents. Adolescence, 29, 781-804.

Manfra, L., \& Winsler, A. (2006). Preschool children's awareness of private speech. International Journal of Behavioral Development, 30, 537-549.

Montero, I., \& De Dios, M. J. (2006). Vygotsky was right: An experimental approach to the relationship between private speech and task performance. Estudios de Psicología, 27(2), 175-189.

Moreira, K. (2012). Categorización e inferencias en la infancia: Evaluación del desarrollo conceptual a través de la tarea de formación de conceptos artificiales Vygotski-Sakharov (Tesis de maestría inédita). Facultad de Psicología, Universidad de la República, Montevideo, Uruguay.

Sánchez-Medina, J. A., Alarcón-Rubio, D., \& Mata-Benítez, M. (2009). Private Speech beyond Childhood: Testing the Developmental Hypothesis. En A. Winsler, C. Fernyhough, \& I. Montero (Eds.), Private speech: Executive functioning, and the development of verbal self-regulation (pp. 188-197). New York: Cambridge University Press.

Smagorinsky, P. (1998). Thinking and Speech and Protocol Analysis. Mind, Culture and Activity, 5(3), 157-177.

Towsey, P. M. (2009). More Than a Footnote to History in Cultural-Historical Theory: The Zalkind Summary, Experimental Study of Higher Behavioural Processes, 
and "Vygotsky's Blocks". Mind, Culture, and Activity, 16(4), 317-337. doi:10.1080/10749030903086009

Vygotski, L. (1995). Pensamiento y lenguaje. Barcelona: Paidós.

Vygotski, L. (2000). Obras Escogidas III. Madrid: Visor.

Wertsch, J. (1991). Voices of the mind: A sociocultural approach to mediated action. Cambridge: Harvard University.

Winsler, A, Diaz, R., \& Montero, I. (1997). The role of private speech in the transition from collaborative to independent task performance in young children. Early Childhood Research Quarterly, 12, 59-79.

Winsler, A. (2009). Still taking to ourselves after all these years: A review of current research on private speech. En A. Winsler, C. Fernyhough, \& I. Montero (Eds.), Private speech: Executive functioning, and the development of verbal selfregulation (pp. 3-41). New York: Cambridge University Press.

Winsler, A., Fernyhough, C., McClaren, E. M., \& Way, E. (2005). Private Speech Coding Manual. Recuperado de http://winslerlab.gmu.edu/PSCodingManual.pdf Winsler, A., \& Naglieri, J. (2003). Overt and Covert Verbal Problem-Solving Strategies: Developmental Trends in Use, Awareness, and Relations With Task Performance in Children Aged 5 to 17. Child Development, 74(3), 659-678.

\section{Formato de citación}

Hontou C. \& Moriera K. (2017). Habla privada en resolución de problemas en adolescentes de 15 años. Psicología, Conocimiento y Sociedad, 7(2), 76-97. Disponible en: http://revista.psico.edu.uy/ 